\title{
Comparative Analysis of Information Exchange in Online and Print Journalism
}

\author{
Kim Chol Hwan', Ho Chol Ung ${ }^{2}$ \\ ${ }^{1}$ College of Literature, Kim Il Sung University, Pyongyang, DPRK \\ ${ }^{2}$ faculty of education, Kim Il Sung University, Pyongyang, DPRK
}

*Corresponding Author: Kim Chol Hwan, College of Literature, Kim Il Sung University, Ryongnamdong, Taesong District, Pyongyang, DPRK

\begin{abstract}
This paper is designed to make the online journalism peculiar and advantageous by comparing it with that in print service.

In this manuscript, online and print news were comparatively analyzed in terms of direction, speed and scope of information exchange and information expression form, as well as its Transmission Avenue and management.
\end{abstract}

As a result, it was found that, in the aspect of the direction of information exchange, online news is provided in many ways. It was also found that, regarding the speed and scope of information exchange, online news is more rapid than print news and the former is broader than the latter in terms of the scope of information exchange.

As far as the expression mode of information is concerned, online news is characterized by multimedia and hypertext and the transmission avenue of information is diverse.

And, in regard to the management of information exchange, online news focuses mainly on the management of its participants and information content.

Keywords: Online Journalism, Rapidity, Extensiveness, Information Exchange, Print Journalism

\section{INTRODUCTION}

The right comparative analysis of information exchange in online and print news services is of great significance in enhancing the might and impact of the online journalism by positively displaying the characteristics and advantages of the online journalism.

As is well known, Internet and online platforms are playing an extremely important role in contemporary society. KajaScheliga and SaschaFriesike write as follows in their theoretical/research paper entitled «Setting up crowd science projects.»

«Crowd science is scientific research that is conducted with the participation of volunteers who are not professional scientists. Thanks to the Internet and online platforms, project initiators can draw on a ... large number of volunteers.»( KajaScheliga and SaschaFriesike et al., 2016)

In the book called «Excellence in Online Journalism», David A. Craig noted about making excellence of online journalism moving target.(David A. Craig, 2011)

«It does not take long to find examples of poorly done online journalism or to find critics eager to point those examples out. But this book is based on the belief that it is more valuable for students preparing for the field to see good examples and to think about what excellent work looks like. Like the technologies that support it, the craft of online journalism is evolving quickly. That makes excellence a moving target.»

Therefore, our topic is to clarifty the characteristics of the online news serve through the comparative analysis of the online and print news services in information exchange and prove them in depth.

In the past, some issues relating to the characteristics of the online news in information exchange were mentioned in several books and theses, but they were not systematized and explained in detail. 
Christian Burggraaff and Damian Trilling wrote as follows in their article entitled «Through a different gate: An automated content analysis of how online news and print news differ.»

«We showed that there are visible differences between online and print news in terms of news values. These differences are related to the different routines used in the two news environments. While this article provides a rather general overview and can be seen as a first step in the quantitative analysis of news values in online and offline news, further (also qualitative or comparative)research is necessary. For future research it may be fruitful to also investigate the actual amount of readers in order to shed further light on the use of website metrics.» (ChristiaanBurggraaff et al, 2017)

As they noted, research into the differences between online and print news are on the table and the importance of qualitative or comparative research is focused on.

This article focuses on the theoretical clarification and intends to guarantee its persuasiveness and logic with the backup of examples.

In this thesis, the features of online journalism are clarified by comparatively analyzing the online and print news services in the aspects of direction, speed, scope, expression form and transmission route and management of information exchange etc.

\section{COMPARISON IN THE DIRECTION OF INFORMATION EXCHANGE}

There are a series of differences between the online and print services.

Above all, they are different in the direction of information exchange.

The print news service makes information exchange broadly in one way, whereas the online one conducts it in many ways.

In the print service, information exchange is often conducted in such a way that reporters and editors deal with readers, listeners and viewers on their own initiative, while the latters receive information and respond to it.

In the online service, however, it is conducted in a range of ways.

Journalists and editors in that service and users exchange various pieces of information.

The former can diffuse news and all other kinds of information promptly among usersby means of emails or chat rooms.

And the latter can rapidly transmit online to the former their own views about the information they have received, new information they have, their opinions related to the operation of the news homepage, etc.

Stefano Calzati also comparatively analyzes the difference between print media and online media in his original article entitled "Chinese domestic tourism, the blogosphere, and travel writing: Assessing the literary and political status of Chinese travelogues in print and online."

"The analysis shows that print travelogues are very politicized, thus departing sensibly from the tradition of the genre. By contrast, travel blogs, despite being published online, are chiefly focused on light topics or the appreciation of the country's natural beauties ..."(Stefano Calzati, 2016)

Their immediate responses to information enable the online journalists, editors and users to build up close connection.

In the course of information exchange the reporters and editors of online journalisms can quickly grasp the matters of users' concern, their views about new facts and events and their opinions about the content and form of news service and, based on this, edit presentations and adopt their content and form in line with the demands and intentions of the masses, thereby representing, leading and organizing fair public opinions.

Jim Macnamara writes about the influence of PR on media content in his article entitled "The Continuing Convergence of Journalism and PR: New Insights for Ethical Practice From a ThreeCountry Study of Senior Practitioners»: The influence of public relations (PR) on media content has been shown to be substantial, and research indicates that it is growing through new media practices." (Jim Macnamara, 2015) 
In the online journalism, information exchange is made between journalists, editors and users.

Online journalists and editors exchange information online with their counterparts in other news services, as well as with their fellows in the same service, and cooperate with each other and deepen mutual understanding as they share views and opinions about different matters and facts.

Users post their views about the new facts and events published on homepages on the bulletin boards or make them public in forums and discuss matters of concern with other users.

For example, as they watch World Cup soccer games on a homepage, users exchange their views on football with each other.

In online journalism, the diffusers and receivers of information exchange information in a threedimensional way.

It is a method of exchanging information about any one issue by one medium among journalists or editors and users and it can be said to be an exchange that fully embodies the features of computer network.

Journalists, editors and users can exchange their views or opinions about any facts or events by means of chat rooms and bulletin boards.

Through such media, the relationship of 3-D information exchange can be formed between reporters and editors, between journalists and users and between users, irrespective of regions, places and time.

In this way, in online journalism, information is exchanged in different ways to enhance the efficiency of information.

\section{COMPARISON IN TERMS OF SPEED AND SCOPE OF INFORMATION EXCHANGE}

\subsection{Rapidity}

The speed of information exchange in the online service is incomparably faster.

Generally, guaranteeing speediness is one of the principles required by publications.

Great rapidity in information communications should be provided. Only then, can the publications guarantee the value of hot news as they mainly consist of them in the contents of editing.

Incorporating computer, telecommunications and telegraph, online journalism can provide people with latest news earlier than other mass media.

The rapidity of online journalism finds its expression in two ways.

First of all, it reports on another news or events instantly on the network.

It is the great advantage of online journalism to promptly load important events that are going on at home and abroad.

Next, it rapidly updates its editing materials.

As soon as important events happen, it sends them out in no time and updates editing materials as fast as possible for the sake of its rapidity. Its updating speed is comparatively high.

As far as the traditional news media is concerned, the period of updating the newspaper contents is from one day to one week in general and radio and TV programmes one day. However, online journalism incessantly upgrades some news items by minutes and by seconds.

However, the rapidity of online journalism also poses some problems.

Some journalists and editors immediately put facts and incidents on the net after they happened to save time without any analysis or consideration, which allow a lot of junk information to exist and adversely affect the identification of necessary information.

And certain facts and incidents are immediately put on the net in spite of its future uncertainty and are supplemented and adjusted after changes afterwards, which also affect the reliability of journalism.

Therefore, it is important to properly choose and analyze facts, while maintaining the rapidity of online journalism. 
The great rapidity of online journalism is related to its means of transmission.

The traditional news media are subject to some restrictions in terms of transmission speed. Transmission means of the newspaper with paper as a medium is the post and delivery. Therefore, its transmission speed is limited to transmission vehicle, sales service and postman's role.

Hence, online media's role is being enchanced. Colin Sparks et al, concludes as follows in their article entitled «The impact of digital media on newspapers: Comparing responses in China and the United States.»

«There is no doubt that, in the developed world, the diffusion of the Internet and other digital technologies has provoked a major crisis for newspapers. ... newspapers are losing their unique role of providing detailed news and information .... .»

Radio and TV broadcast transmit their information to some regions by means of wireless electronic signals, thus being restricted by receiver regions. Signals can be transmitted when auxiliary relaying means needs to be where the signals fail to reach for their successful transmission. These factors influence the transmission speed of information.

Optical fiber communication line is mostly used as the transmission means of online journalism. Information can reach any necessary regions in the world in a flash via optical fibre cables, which is a firm guarantee for the rapidity of online journalism.

Along with this, the simplicity of the online journalism processes provides an important guarantee for ensuring its rapidity.

As far as the traditional news media processes are concerned, newpaper processes comprise coverage, writing, editing, printing and distribution; radio or TV broadcast proceses constitute coverage, writing, editing and progress. But online journalism needn't print or distribute like newspapers and progress like radio or $\mathrm{TV}$, so it can cut the report timeshort.

Every process of traditional news media also consumes much time.

Journalists engaged in this kind of work devote so much time to coverage, writing of manuscripts, shooting, recording and others. On receiving the manuscript, the editorial staff spends too much time on editing of manuscripts, sound editing, developing of photos, electronic image editing and printing.

Some news items require further survey of the events and thus need more time for the addition of such materials as background, characters, photos, sound and images.

In this way, it takes much time in traditional news media processes for the news to reach receivers since the facts or events have occured.

On the contrary, those of online journalism cover and edit the events, send the information to the editorial staff via the network, and search required materials on the network database. This makes it possible to save much valuable time and provide news materials as soon as possible.

When important facts or events happen, they may be broadcast live by radio and TV. But they have to make a lot of detailed arrangements for live broadcasting and their machinery are very complex.

However, online journalists employ such digital means as portable computers and digital cameras so that they can report on the ongoing events directly via the network.

When an event happens, so to speak, online journalism enables receivers to listen to and watch the report on the event in real time on the network.

Online journalism can receive and send news scripts at any time. This is an important guarantee for its rapidity.

In case of newspapers, it is needed to issue an extra edition or re-edit as a whole in order to add another news to it after finishing editing.

In this case, on radio or TV, it needs to lengthen the time of carrying the scripts or stop regular programmes and report on sudden news or events.

But, on the network, it is free to incessantly receive and send scripts all day without any restrictions. 
A great rapidity of online journalism is related to such articles widely posted on the homepages, such as those on scrolls, and real-time reports, both of which help ensure the rapidity.

Scroll report is the article on the recent news of importance presented by the scroll bar.

On collection of a hot news, it is loaded on the homepage in two ways: one is to erase the existing content and post it and the other is to post it by use of the scroll bar.

The scroll bar is put where the attention is easily paid and runs through the contents horizontally or vertically.

Scroll report is the way of presenting latest news by means of the scroll bar and is of significance in raising the rapidity of online journalism.

Live broadcast indicates instant presentation of articles on the ongoing news or events.

Contrary to that by radio or TV, live broadcast by online journalism is quicker in presentation of articles, richer in their expression and more extensive in the range of communications than the former.

It can be carried out by using various forms, such as characters, sound, images, multimedia and so on.

Journalists can broadcast live instantly in the forms of characters, sound, images and multimedia on the network by using computers, mobile phones, cameras and others on the spot.

Among them live broadcast by characters is peculiar to the network.

Live broadcast by characters is the presentation of the news by characters while journalists cover the events on the spot.

It is posted on the special live broadcast webpage.

Compared with live broadcast on TV, it fully records the progress and details of any events and preserves them for the long duration, not to speak of its great rapidity. It also has the advantage for online users to read in an instant or in a prolonged time.

\subsection{Extensiveness}

Online journalism is extensive in the communication of information.

It indicates that online journalism is extensive in the contents of information communications and also in their range.

It is extensive in the contents of information communications.

Traditional news media are limited in their space or broadcast time, resulting in the relative restriction of their information volume.

As the newspaper space is limited, the number of its characters or photos available is fixed.

Radio or TV broadcast the restricted amount of information within a fixed time.

For this reason, it fails to carry a large amount of programmes and their choice poses an important problem.

In addition to this, once its programme has just been broadcast, it can never be repeated. That's why it gives a full explanation. It is, after all, subject to restriction on broadcasting many programmes.

In the interim, as TV programme is broadcast on certain channels, it is limited by the choice of its programmes.

From this point, editors of the traditional news media attach great importance to the choice and presentation of materials.

A news agency in a country sends 70 percent of recieved information to a news medium, in which only two percent of them are chosen for broadcast.

Online journalism media, however, have a mammoth memory capacity of information, thus providing deliverers with an infinite scope.

The online information volume further exceeds the biggest library in the world and at present is formally recognized by humans as the biggest and most comprehensive information soruce base. 
That online journalism is extensive in its contents of information communication is shown in three aspects.

Online journalism is extensive in its huge amount of information communications.

In the online journalism media, web pages are super linked to transmit all the necessary contents since they are limited in terms of transmission time.

The online journalism media are not affected in content transmission, so they can transmit such huge amount of contents. At present, the number of news items communicated by allegedly big online journalism media worldwide amounts to more than 1000 on a daily basis.

Online journalism is also extensive in its huge amount of data communications relating to relevant news.

In the online journalism media, when reports on certain facts and events are carried, various data relating to them can be extensively accompanied.

Newsapapers and other news media send out necessary news due to limitations of space or time and limit the communication of relavant data.

Online journalism media, however, carry a variety of data together with main news relating to them, helping receivers understand them.

Online journalism is also extensive in the huge amount of storage data presented.

Online journalism media can store all the data already presented historically. For instance, in case it is a homepage of a traditional newspaper, all the newspapers from the first issue up to now can be stored in digital form. Therefore, that newspaper's homepagecan be said to be a huge network information database.

Alain Peter et. al. emphasizes the importance of digitization of newspaers in their article entitled «Power interplay and newspaper digitization: Lessons from the Pengpai experiment»

«In a matter of 2 years, Pengpai has become a popular national digital news outlet among a middleaged, educated and urban public. Its readership is four times larger than the former DongfangZaobao ... . Undeniably, the digitization process has helped the newsroom gain influence.» (Alain Peter et. al., 2017)

Today manay online journalism media offer data retrieval service. Through this function, online journalism receivers can conveniently retrieve and browse news and data announced in relevant times.

Online journalism is extensive in the range of information communications.

The traditional news media are limited in this regard. The distribution of newspaper requires employing various vehicles, so it is restricted in its range of distribution and in case of distribution to other countries, it may take some five days to the maximum.

Radio or TV broadcast using electric wavesneed to set up relay stations and resolve many technical problems to improve their quality of waves so as to reach any places of the earth.

But the communication scope of online journalism covers wherever network is readily available, namely, all over the world. The network is available in every country and region at present.

The network enables any one to freely exchange information with any other person at any time and in any place. It is not affected by time, channel, weather and national border.

Accordingly, humans can keep themselves up to date with any news from every country in good time via online journalism media and exchange information freely with different news media and people of the world and communicate with them.

As seen above, the rapidity and extensiveness of the information communications mainly feature the online journalism.

\section{COMPARISON IN EXPRESSION MODE AND Transmission ROUTE OF INFORMATION COMMUNICATION}

Next, online and print news services are different in terms of the mode of expression of information and the avenue of its communication. 
In the print service, different modes of expression are adopted according to the content of information, and they are simple comparatively.

Newspapers exchange information mainly by means of letters and visual aids, radio stations by sound and TV stations by sound and image.

Online journalism, however, can employ the modes of expression of information of all other information media, while exchanging information by means of multimedia and hypertext.

In online journalism, letters, pictures, photos, sound, animations and other various expression forms are used to store, to express and to communicate information.

The multimedia characteristics of online journalism in terms of information expression form have various advantages.

For one thing, the strength is that the online journalism media can use all the information in different expression forms.

Information in the various expression forms including letters, sound and images is freely circulated on the network.

For example, a newspaper homepage can contribute to vividly reproducing reality by presenting sound and images as well, while focusing mainly on letter information.

For another, the strength is that users can freely select the expression forms of information.

Since online journalism media make use of multimedia forms to receive, express and communicate information, they provide users with conditions to choose information with any expression forms.

Users can choose and read letter-only information without sound, information with sound and images, or information in which photos and letters are combined with other different expression forms.

The current sound and image information available on the internet are less qualified due to various technological problems. They also lack the amount of data edited and the number of simultaneous readers.

The incident of the World Trade Center in New York on 11 September 2001 is a quintessential example. At that crisis, news sites were unable to deal with the sudden increase in demand for up-todate coverage. (Garrett Monaghan and Sean Tunney, 2010)

With the development of IT, the frequency band of the internet will be increased by hundreds of times and internet news information will exist mainly in the form of multimedia, not in the characteroriented form in the future.

Online journalism is characterized by hypertextualization in the form of information expression.

Unlike an ordinary text in which it is read from the first page sequentially, a hypertext is browsed freely moving from any page to another page of the text. In the hypertext, once you click on an element (word, sentence or image), you move on to another page linked to that element. Then the page linked can be any page available on any computer on the internet.

Hypertextualization of information expression form in online journalism enables us to retrieve and browse necessary information in time, provides a range of information relating to relevant data and permits prompt uploading, deletion and correction of edited data.

In print journalism, the avenue is comparatively limited to readers' gatherings, readers' letters, opinion polls etc.

In online journalism, however, it is varied, such as e-mails, electronic bulletin boards, chat rooms, mobile phones, video meetings, online investigations etc.

Diversity in terms of information transmission route is of great significance in that it enables users to present their own views or opinions concerning relevant news items on the network in time, expands and intensifies the information exchange between online journalism institutions and users and promotes online journalism in keeping with the demands and mindset of popular masses.

Charlie Beckett and Mark Deuze mentioned the trend of a new networked news media in their article entitled "On the Role of Emotion in the Future of Journalism." 
The trend is clear: toward a more mobile, personalized, and emotionally driven news media.(Charlie Beckett and Mark Deuze, 2016)

Jacob L. Nelson and Jame G. Webster wrote in their article entitled "The Myth of Partisan Selective Exposure: A Portrait of the Online Political News Audience".

"The idea that a person's ideology may affect how they engage with a news story has been explored in the past. We hope that our findings will encourage scholars to put more of their efforts into investigating this relationship going forward."( Jacob L. Nelson and Jame G. Webster, 2017)

As science and technology develop and people's requirements grow, the channel of information exchange will get more diversified.

\section{COMPARISON IN THE MANAGEMENT OF INFORMATION EXCHANGE}

Online and print news services are also different in the management of information exchange.

In the print one, information exchange is undertaken by a particular department. And as far as its general management is concerned, the department asks readers, listeners and viewers to write articles, revises their letters and articles to carry in relevant information media or arranges such gatherings as readers' meetings to inform related departments of the matters.

But the online service mainly manages the participants in information exchange and the content of information.

Information exchange on the internet is not confined to one country but covers the whole world.

Therefore, broad sections of people around the world participate in information exchange in online journalism and, based on this prerequisite, the online information exchange is analyzed.

Above all, what is important in the management of information exchange in online journalism is the management of participants in information exchange.

Various sections of people take part in online information exchange. Most websites are not exact in demand comparatively in user registration as they want to increase the number of users and encourage their active participation in information exchange, but this may lay obstacles to its management.

Therefore, a proper management of participants is imperative.

It is, first of all, necessary to manage their identities and qualifications.

The manager ascertains their identities and categorizes them, based on the information they offered. By doing so, the manager can register learned persons, good debaters and critics and the like in the special user management database and get familiar with these users' information exchange.

If necessary, the manager may get in direct contact with each of them to enable them to have proper influence on information exchange.

Along with the management of their identities and qualifications, it is also important to motivate them to take active participation in information exchange.

This can be done by keeping close contact with users to lead them to publish good articles on relevant issues and post them online, so that they should be active in information exchange.

Moritz Büchi and Florian Vogler argue about the way participants participate in accordance with the increase of Internet use in their article entitled "Testing a Digital Inequality Model for Online Political Participation."

"The level of individuals' Internet experience and skills codetermines their online political participation.

Based on these empirical findings, we argue that public policies aimed at increasing democratic participation in the digital society may need to complement ... through education.

Access to and use of the Internet have become requirements for full participation in the digitally enabled state."

They further claim that policies need to focus on effective use of the Internet and make it permanent activity. 
The management of the content of information exchange is also important in that of information exchange in online journalism.

It includes the making of rules, examination and screening, and selection and recommendation.

The rule making covers the making of standards of examination and screening of content and rules of selection.

Each homepage has its own standards of examination and screening and rules of selection.

The examination and screening are technical examination and screening by examiners.

As the content of online articles is complex and large in number, the screening cannot be done only by examiners in time.

Therefore, certain key words should be given to those articles and technical retrieval be carried out by means of filtering software, so that examiners can examine them, based on relevant provisions.

The selection and recommendation are for the content manager to select refined articles in time in the process of reading and examining users' articles, and recommend them for online publication.

The selected articles should, of course, be matters of readers' concern and worthy of reporting and publicity.

These can also be recommended to other homepages, including one's own.

We should make active efforts to improve the management of information exchange with a good understanding of the characteristic features of this exchange, one of important processes in online journalism.

\section{CONCLUSION}

The print news service makes information exchange broadly in one way, whereas the online one conducts it in many ways.

The speed of information exchange in the online service is incomparably faster than that in the print one.

The journalists and editors of print news services are unable to exchange information with readers, listeners and viewers as fast as online services due to the limitations of place, time and forms of exchange.

But in online journalism, when a news item is posted on a homepage, for example, users can send their opinions to relevant journalists by means of online communication means like e-mails immediately after they see it.

In online journalism, a far wider range of information exchange can be conducted than in the print service. Here, information can be exchanged unaffected by place and time through various media and, therefore, broad sections of people can take an active part in information exchange.

Online journalism can employ the modes of expression of information of all other information media, while exchanging information by means of multimedia and hypertext.

Online and print news services are different in the management of information exchange.

In the print one, information exchange is undertaken by a particular department. And as far as its general management is concerned, the department asks readers, listeners and viewers to write articles, revises their letters and articles to carry in relevant information media or arranges such gatherings as readers' meetings to inform related departments of the matters raised there.

But the online service mainly manages the participants in information exchange and the content of information.

\section{ACKNOWLEDGEMENTS}

The authors would like to thank Prof. and PhD Sin Yong Ho and Prof. and PhD Ko Yong Kuk for their invaluable contribution to the data collection and academic assistance. 


\section{REFERENCES}

[1] Jim Macnamara, (2015), The Continuing Convergence of Journalism and PR: New Insights for Ethical Practice From a Three-Country Study of Senior Practitioners, Journalism \& Communication Quarterly, Vol. 93(1) 118-141

[2] ChristiaanBurggraaff, Damian Trilling, (2017), Through a difference gate: An automated content analysis of how online news and print news differ, Journalism, 1-18

[3] Alain Peter et al, (2017), Power interplay and newspaper digitization: Lessons from the Pengpai experiment, Global Media and China, Vol. 1(4) 497-510

[4] Moritz Buchi and Florian Volger, (2017), Testing a Digital Inequality Model for Online Political Participation, Sociological Research for a Dynamic World, Volume 3 1-13

[5] Charlie Beckett and Mark Deuze, (2016), On the Role of Emotion in the Future of Journalism, Social Media+Society, 1-6

[6] Jacob L. Nelson and Jame G. Webster, (2017), The Myth of Partisan Selective Exposure: A Portrait of the Online Political News Audience, Social Media+Society, 1-13

[7] Stefano Calzati, (2016), Chinese domestic tourism, the blogsphere, and travel writing: Assessing the literary and political status of Chinese travelogues in print and online, Global Media and China, Vol.1(3), $168-185$

[8] Colin Sparks et al., (2016), The impact of digital media on newspapers: Comparing responses in China and the United States, Global Media and China, Vol. 1(3) 186-207)

[9] KajaScheliga and SaschaFriesike, 2016, Setting up crowd science projects, Public Understanding of Science, $1-20$

[10] David A. Craig, (2011). Excellence in Online Journalism, SAGE Publications, Inc.

[11] Garrett Monaghan and Sean Tunney, (2010), Web journalism://a new form of citizenship?, Sussex Academic Press

Citation: Kim Chol Hwan, Ho Chol Ung. "Comparative Analysis of Information Exchange in Online and Print Journalism". International Journal of Media, Journalism and Mass Communications (IJMJMC), vol 4, no. 3, 2018, pp. 1-10. doi:http://dx.doi.org/10. 20431/2455-0043.0403001

Copyright: (ㅇ 2018 Authors. This is an open-access article distributed under the terms of the Creative Commons Attribution License, which permits unrestricted use, distribution, and reproduction in any medium, provided the original author and source are credited. 